\title{
PVP2013-97221
}

\section{DRAFT: LINEAR MATCHING METHOD FOR PARAMETRIC STUDIES OF WELDMENTS CREEP-FATIGUE ENDURANCE}

\author{
Yevgen Gorash \\ Department of Mechanical \& Aerospace Engineering \\ University of Strathclyde \\ James Weir Building, 75 Montrose Street \\ Glasgow G1 1XJ, United Kingdom \\ Email: yevgen.gorashestrath.ac.uk
}

\author{
Haofeng Chen* \\ Department of Mechanical \& Aerospace Engineering \\ University of Strathclyde \\ James Weir Building, 75 Montrose Street \\ Glasgow G1 1XJ, United Kingdom \\ Email: haofeng.chenestrath.ac.uk
}

\begin{abstract}
This paper presents parametric studies on creep-fatigue endurance of the steel AISI type $316 N(L)$ weldments defined as types 1, 2 and 3 according to R5 Vol. 2/3 Procedure classification at $550^{\circ} \mathrm{C}$. The study is implemented using the Linear Matching Method (LMM) and based upon previously developed creepfatigue evaluation procedure considering time fraction rule. Several geometrical configurations of weldments with individual parameter sets, representing different fabrication cases, are developed. For each of configurations, the total number of cycles to failure $N^{\star}$ in creep-fatigue conditions is assessed numerically for different loading cases. The obtained set of $N^{\star}$ is extrapolated by the analytic function dependent on normalised bending moment $\tilde{M}$, dwell period $\Delta t$ and geometrical parameters. Proposed function for $N^{\star}$ shows good agreement with numerical results obtained by the LMM. Therefore, it is used for the identification of Fatigue Strength Reduction Factors (FSRFs) intended for design purposes and dependent on proposed variable parameters.
\end{abstract}

\section{NOMENCLATURE}

$\begin{array}{ll}\Delta \sigma & \text { stress range } \\ \varepsilon & \text { strain } \\ \dot{\varepsilon} & \text { strain rate } \\ \Delta \varepsilon & \text { strain range } \\ \omega & \text { damage parameter } \\ t & \text { time }\end{array}$

*Address all correspondence to this author. $t^{*}$

$\Delta t$

E

$\bar{E}$

$\mu$

$N^{*}$

$N^{\star}$

$L^{\star}$

$\tilde{M}$

$\Delta M$

$P$

$I_{X}$

$w$, thk

$\alpha, \beta, \rho$

$R_{1}, R_{2}, R_{3}$

$\delta$

D

$a$

$h_{1}, d_{1}, h_{2}, d_{2}, h_{3}$

$\sigma_{\mathrm{y}}$

$B, \beta$

$p_{0}, p_{1}, p_{2}$

$a_{0}, \ldots, a_{3}, b_{0}, \ldots, b_{3}$ time to pure creep failure

dwell period

Young's (elasticity) modulus

effective elastic modulus

Poisson's ratio

number of cycles to pure fatigue failure number of cycles to creep-fatigue failure residual life normalised bending moment bending moment range normal pressure area moment of inertia width and thickness of plate parameters governing the profile form of type 1,2 and 3 weldments radiuses of weld profile for type 1,2 and 3 weldments correspondingly height of weld profile in type 1 weldment distance between opposite weld surfaces in type 2 weldment width of weld throat in type 3 weldment auxiliary geometrical parameters for type 1 , 2 and 3 weldments correspondingly yield stress R-O model constants coefficients for parent material S-N curve fitting parameters for $N^{\star}$ 


\section{INTRODUCTION}

According to industrial experience, during the service life of welded structures subjected to cyclic loading at high temperature, welded joints are usually considered as the critical locations of potential creep-fatigue failure. This is caused by higher stress concentration, altered and non-uniform material properties of weldments compared to the parent material of the entire structure. Therefore, creep and fatigue characteristics of welded joints are of a priority importance for long-term integrity assessments and design of welded structures. There were attempts to develop analytical tools [1,2] to estimate long-term strength of welded joints under variable loading. However, residual life assessments are frequently complicated and inaccurate because of complex material microstructure and too many parameters affecting the strength of welded joints. In view of the complexity of a unified model development for the assessment of creep-fatigue strength, there are a limited number of existing analytical approaches, but none of which are able to account for all of weldment parameters mentioned above. Thus, long-term strength of weldments is a wide research area, which requires some unified integral approach able to improve the life prediction capability for welded joints. The most comprehensive overviews of studies devoted to investigation of influence of various parameters on fatigue life of welded joints are presented in [1,2]. However, the influence of creep on residual life is not investigated in these works.

This paper presents further extension of a latest developed approach [3], which includes a creep-fatigue evaluation procedure considering time fraction rule for creep-damage assessment and a recent revision of the Linear Matching Method (LMM) to perform a cyclic creep assessment [4]. The applicability of this approach to a creep-fatigue analysis was verified in [3] by the comparison of FEA/LMM predictions for an AISI type $316 \mathrm{~N}(\mathrm{~L})$ steel cruciform weldment at $550^{\circ} \mathrm{C}$ with experiments by Bretherton et al. [5, 6] with the overall objective of identifying fatigue strength reduction factors (FSRF) of austenitic weldments for further design applications. An overview of previous modelling studies devoted to analysis and simulation of these experiments [5,6] is given in [3]. The parametric study presented in this paper is based on the research outcomes given in prior work [3] successfully validated by matching the basic experiments [5, 6]. Thus, exactly the same assessment approach is applied to parametric studies of the weldment geometry in order to assess the effect on the predicted residual life.

Another outcome of the previous work [3] is the formulation of an analytical function for the total number of cycles to failure $N^{\star}$ in creep-fatigue conditions, which is dependent on normalised bending moment $\tilde{M}$ and dwell period $\Delta t$. This function $N^{\star}(\tilde{M}, \Delta t)$ matches the LMM predictions with reasonable accuracy and is used for the investigation of $\Delta t$ influence on the FSRF. Therefore, the effect of creep on long-term strength of type 2 dressed weldments (according to the classification in R5 Vol. 2/3 Procedure [7]) is taken in to account.
Apart from operational parameters $(\tilde{M}$ and $\Delta t)$, it is necessary to investigate the influence of a weld profile geometry on creep-fatigue strength within a parametric study. The introduction of geometrical parameters into the function $N^{\star}(\tilde{M}, \Delta t)$ allows the calculation of the FSRF as a continuous function able to cover a variety of weld profile geometries including type 1,2 and 3 in dressed, as-welded and intermediate configurations.

\section{PARAMETRIC MODELS OF WELDMENTS Geometrical relations}

It has been indicated [1] that one of the most critical factors affecting the creep-fatigue life of a welded joint is the consistency of the cross-sectional weld geometry. The simplified weld profile is usually characterised by the following geometric parameters [1]: plate thickness, effective weld throat thickness, weld leg length, weld throat angle, and weld toe radius. Usually, the weld profile is assumed to be circular for type 1, circular or triangular for type 2, and triangular for type 3 weldments with fillets on toes connecting with parent plates. A vast quantity of researches reviewed in [1,2] has been devoted to investigation of effects produced by geometrical parameters on residual life.

In the present study, the geometry of the weld profiles for type 1, 2 and 3 weldments is more completely specified in order to investigate their as-welded, dressed and intermediate configurations. The basis of the parametric models for type 1 and 2 weldments shown in Fig. 1 are the sketches of the weldment specimens produced by the Manual Metal Arc (MMA) welding and reported in [5]. The type 1 weldment specimen contains a double-sided V-butt convex-fillet weld, and the type 2 weldment specimen contains 2 symmetric double-sided T-butt cruciform concave-fillet welds. The basis of the parametric model for type 3 weldment shown in Fig. 1 1 are the sketches of weld profiles and corresponding regulations from British Standards [8, 9] for the weldment, which contains a root gap between the parts to be joined. The type 3 weldment specimen contains 2 symmetric double-sided T-butt cruciform mitre-fillet welds.

The parent material for the manufacturing of all specimens are continuous plates of width $w=200 \mathrm{~mm}$ and thickness $t h k=$ $26 \mathrm{~mm}$ made of the steel type AISI $316 \mathrm{~N}(\mathrm{~L})$. The typical division of the weld into three regions is adopted here analogically to [3] including: parent material, weld metal and heat-affected zone (HAZ). It should be noted that the HAZ thickness is assumed to be $3 \mathrm{~mm}$ based on the geometry given in [5]. These 3 regions have different mechanical properties described by the following material behaviour models and corresponding constants at $550^{\circ} \mathrm{C}$ in [3] for the FEA with the LMM:

- Elastic-perfectly-plastic (EPP) model for the design limits as a result of shakedown analysis;

- Ramberg-Osgood (R-O) model for the plastic and total strains under saturated cyclic conditions; 


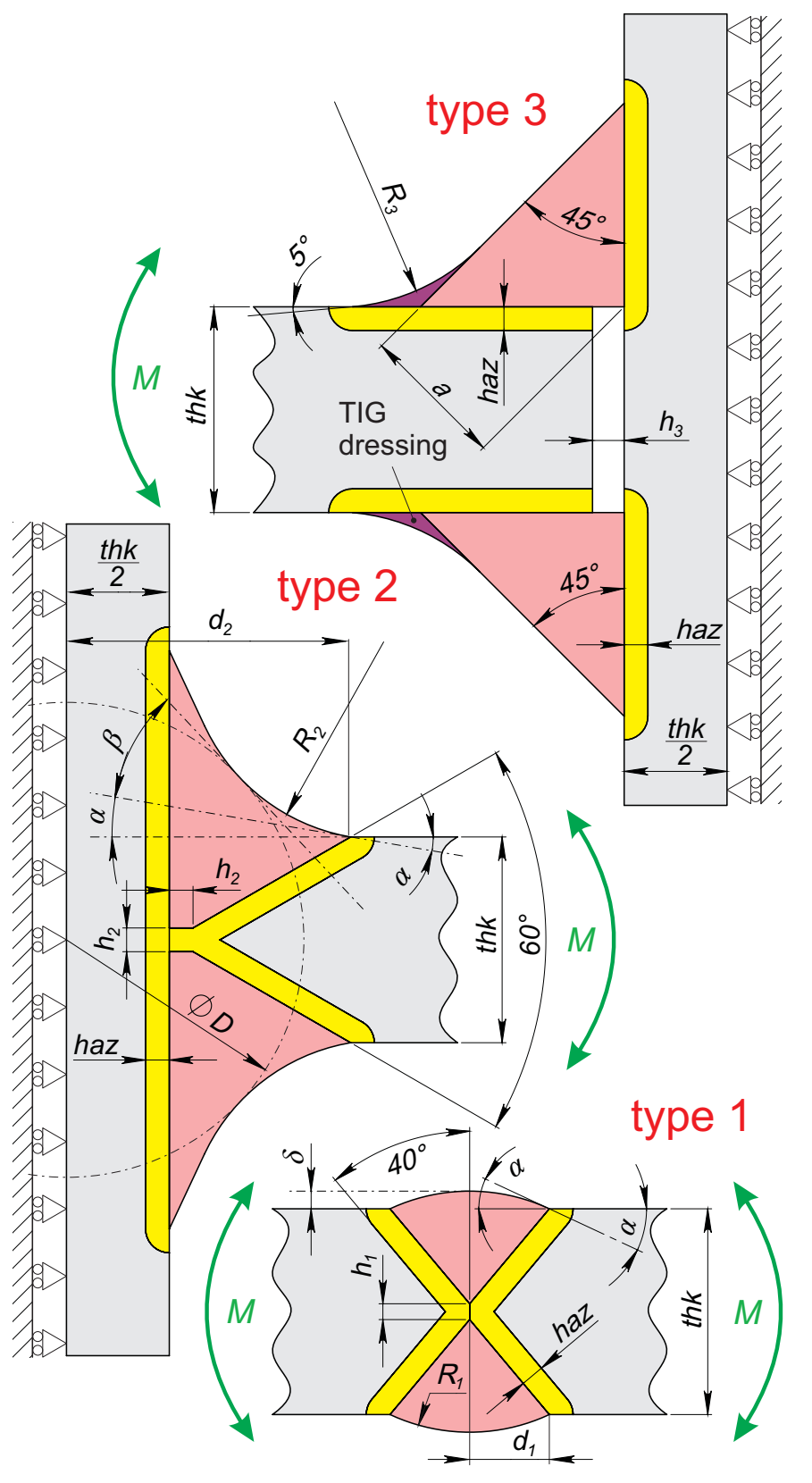

FIG. 1 WELD PROFILE GEOMETRIES OF TYPES 1, 2 AND 3 WELDMENTS ACCORDING TO R5 [7]

- $\mathrm{S}-\mathrm{N}$ diagrams for the number of cycles to failure caused by pure low-cycle fatigue (LCF);

- Power-law model in "time hardening" form for creep strains during primary creep stage;

- Reverse power-law relation for the time to creep rupture caused by creep relaxation during dwells;

- Non-linear diagrams for creep-fatigue damage interaction for the estimation of total damage.
The profile geometry of type 2 weldment is comprehensively characterised by one of two pairs of parameters: (1) independent parameters $(\alpha$ and $\beta$ ), which are not dependent on a plate thickness $t h k$, and (2) technologically controlled parameters $\left(R_{2}\right.$ and $D$ ), which change their values with a change of plate thickness thk. In parametric relations for strength of type 2 weldments the independent parameters $(\alpha$ and $\beta$ ) are used with a capability of transformation into controlled parameters $\left(R_{2}\right.$ and $\left.D\right)$. As illustrated in Fig. 1, angle $\alpha$ represents a local geometrical nonuniformity caused by a deviation from the tangent condition between parent plate and weld. Angle $\beta$ represents a global geometrical non-uniformity caused by deposition of weld metal connecting the orthogonal part.

The relations between the two parameter pairs $\left(\alpha, \beta\right.$ and $R_{2}$, $D$ ) for a type 2 weldment are formulated using basic trigonometric calculus in conjunction with the thickness of a plate crosssection thk and the corresponding associated parameters $\left(h_{2}\right.$ and $d_{2}$ ) as illustrated in Fig. 11

$$
h_{2}=\frac{t h k}{8.6666} \text { and } d_{2}=\frac{t h k}{2}+h_{2}+\frac{t h k-h_{2}}{2} \tan 60^{\circ} .
$$

The direct transitions are formulated as follows

$$
\begin{aligned}
& R_{2}=\frac{\frac{t h k / 2}{\cos (\alpha+\beta)}-\frac{d_{2}}{\sin (\alpha+\beta)}}{\frac{\sin \alpha}{\sin (\alpha+\beta)}-\frac{\cos \alpha}{\cos (\alpha+\beta)}} \text { and } \\
& D=2 \frac{R_{2} \cos \alpha+t h k / 2}{\cos (\alpha+\beta)}-2 R_{2} .
\end{aligned}
$$

The reverse transitions are formulated as follows

$$
\begin{aligned}
\beta & =\arccos \left[\frac{d_{2}^{2}+(t h k / 2)^{2}-R_{2}^{2}-\left(R_{2}+D / 2\right)^{2}}{-2 R_{2}\left(R_{2}+D / 2\right)}\right], \\
\alpha & =90^{\circ}-\arctan \left(\frac{t h k}{2 d_{2}}\right)-\beta \\
& -\arccos \left[\frac{R_{2}^{2}-\left(R_{2}+D / 2\right)^{2}-d_{2}^{2}-(t h k / 2)^{2}}{-2\left(R_{2}+D / 2\right) \sqrt{d_{2}^{2}-(t h k / 2)^{2}}}\right] .
\end{aligned}
$$

Relations between independent parameter $\alpha$ and controlled parameter $\delta$ for type 1 weldment are formulated using basic trigonometric calculus in conjunction with the thickness of a plate cross-section thk and the corresponding associated parameters $\left(h_{1}\right.$ and $\left.d_{1}\right)$ as illustrated in Fig. 1:

$$
h_{1}=\frac{t h k}{13} \quad \text { and } \quad d_{1}=\frac{t h k-h_{1}}{2} \tan 40^{\circ} .
$$


The direct transition is formulated as follows

$$
\delta=R_{1}(1-\cos \alpha) \quad \text { with } \quad R_{1}=d_{1} / \sin \alpha .
$$

The reverse transition is formulated as follows

$$
\alpha=\arccos \left(\frac{R_{1}-\delta}{R_{1}}\right) \quad \text { with } \quad R_{1}=\frac{\delta}{2}+\frac{d_{1}^{2}}{2 \delta}
$$

Since the geometry of type 3 weldment profile due to mitre fillet is much simpler than the geometry of type 1 and 2 weldments, there are only a few parameters governing this type of geometry. The form of type 3 weld is a isosceles triangle with right angle, as shown in Fig. 1. It is characterised by the weld throat $a$, which should be $(a \geq 0.7 t h k)$ according to the standard [8]. The gap between the welded parts $h_{3}$ should satisfy the requirement $\left(h_{3} \leq 1 \mathrm{~mm}+0.3 \mathrm{~mm} a\right)$, but it shouldn't exceed $4 \mathrm{~mm}$ according to the standard [9].

The fatigue performance of the original type 3 weld profile is quite poor due to significant stress concentration in the weld toe caused by inconsistency of weld profile in $135^{\circ}$. Moreover, the gap between the welded parts decreases the effective crosssection limiting it to the only area of weld metal. For the purpose of the fatigue life improvement, different post weld treatment techniques are applied to the weld toe, as a potential location of failure. TIG dressing was found in [10] to be the best suited post weld treatment for implementation in mass production compared to burr grinding and ultrasonic impact treatment, because of the large improvement observed in the experiments (up to $40 \%$ increase in fatigue strength). Therefore, $R_{3}$ in Fig. 1 is the radius of fillet produced by TIG dressing on the weld toe. The angle of discrepancy for the tangency condition between TIG weld metal and patent plate is $5^{\circ}$, since it is a minimum allowable angle for a finite element in order not to be distorted.

Since the proposed parameters for type 1 and 2 weld profiles are fully convertible, they can be used to characterise different scales of technological dressing of weldments by grinding such as dressed, as-welded and intermediate. Thus, in order to reduce the computational costs, only five configurations of weld profile, listed in Table 1 were chosen for parametric study from among the possible parameter combinations. In case of type 3 weldment, the different scales of TIG dressing are characterised by the parameter ratio $\rho$ between fillet radius and plate thickness:

$$
\rho=R_{3} / t h k
$$

Analogically to Table 1, seven configurations are proposed for the parametric study of type 3 weldment described by the following values of the parameter $\rho: 2.0,1.5,1.0,0.5,0.2,0.1$ and $\rho \rightarrow 0$ corresponding to the undressed configuration.
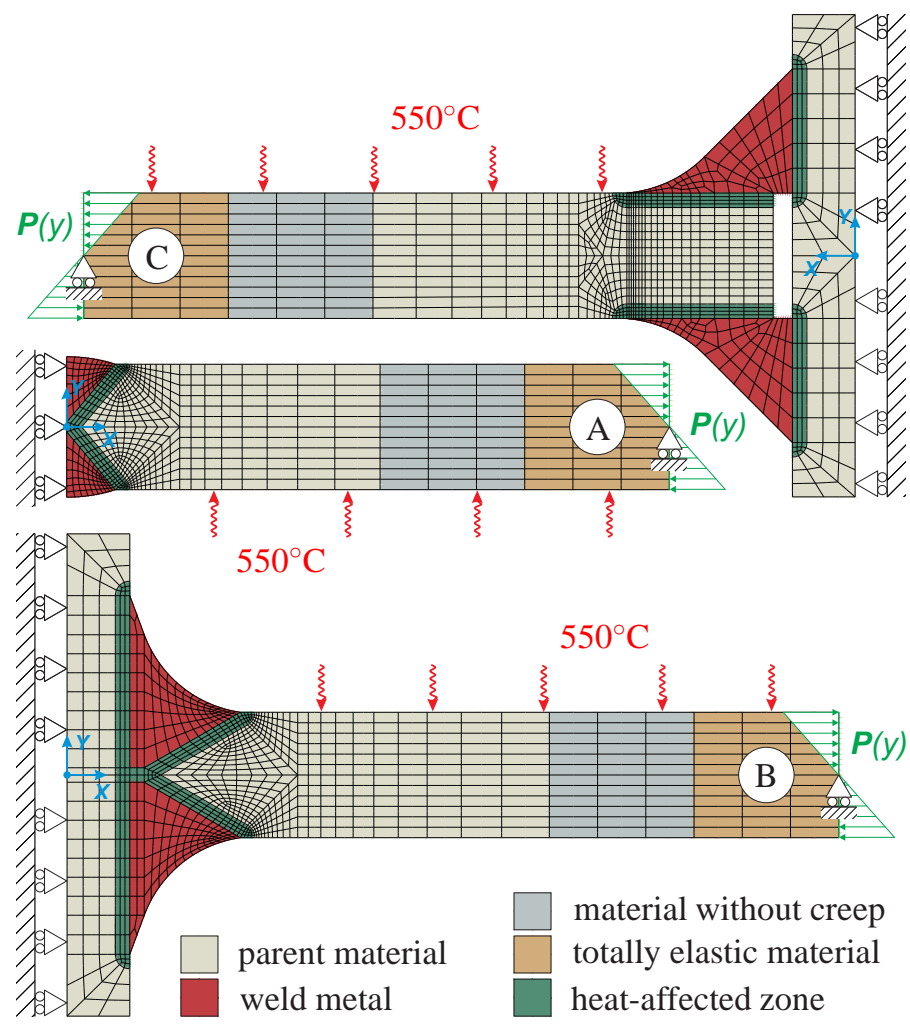

FIG. 2 FE-MESHES FOR TYPE 1 (A), TYPE 2 (B) AND TYPE 3 (C) WELDMENTS WITH LOADINGS

\section{Finite element models}

The FE-meshes for the 2D symmetric models of type 1, 2 and 3 weldments are shown in Fig. 2 assuming plane strain conditions. Each of the FE-meshes includes 5 separate areas with different material properties: 1) parent material, 2) HAZ, 3) weld metal, 4) material without creep, 5) totally elastic material. Introduction of 2 additional material types (material without creep and totally elastic material) representing reduced sets of parent material properties in the location of bending moment application avoids excessive stress concentrations. Both FE-models use ABAQUS element type CPE8R: 8-node biquadratic plane strain quadrilaterals with reduced integration. The FE-meshes for type 1 and type 2 welds consist of 723 and 977 elements respectively. The FE-mesh for type 3 weldwent contains the range of elements from 1008 for Conf. 1 to 908 for Conf. 7 respectively.

Referring to the technical details [5,6] the testing was performed at $550 \pm 3^{\circ} \mathrm{C}$ under fully-reversed 4-point bending with total strain ranges $\Delta \varepsilon_{\text {tot }}$ of $0.25,0.3,0.4,0.6$ or $1.0 \%$ in the parent plate and hold periods $\Delta t$ of 0,1 or 5 hours using a strain rate of $0.03 \% / \mathrm{s}$. For the purpose of shakedown and creep analysis using LMM, the conversion from strain-controlled test conditions to force-controlled loading in the simulations using bending moment $M$ has been carried out and explained in [3]. 


\begin{tabular}{c|l|ccc|ccc}
\hline \hline \multirow{2}{*}{ No. } & \multirow{2}{*}{ Configuration } & \multicolumn{3}{|c|}{ Independent parameters } & \multicolumn{3}{|c}{ Controlled parameters } \\
\cline { 3 - 8 } & & $\alpha$ & $\beta$ & $\alpha+\beta$ & $D$ & $R$ & $\delta$ \\
\hline 1 & Perfectly dressed & 0 & 43.387 & 43.387 & 54.578 & 25 & 0 \\
2 & Typically dressed & 7.745 & 38.382 & 46.127 & 59 & 25 & 0.682 \\
3 & Precisely as-welded & 17.685 & 32.079 & 49.764 & 64 & 25 & 1.566 \\
4 & Typically as-welded & 32.371 & 18.415 & 50.786 & 68 & 40 & 2.923 \\
5 & Coarsely as-welded & 45.177 & 9.6541 & 54.831 & 72 & 60 & 4.189 \\
\hline \hline
\end{tabular}

Another effective analysis technique, successfully employed in [3], was to apply the bending moment $M$ through the linear distribution of normal pressure $P$ over the section of the plate as illustrated in Fig. 2 with the area moment of inertia in regard to horizontal axis $X$ :

$$
I_{X}=w t h k^{3} / 12,
$$

where the width of plate $w=200 \mathrm{~mm}$ and the thickness of plate thk $=26 \mathrm{~mm}$. Therefore, the normal pressure is expressed in terms of applied bending moment $M$ and vertical coordinate $y$ of plate section assuming the coordinate origin in the mid-surface:

$$
P(y)=M y / I_{X} .
$$

\section{STRUCTURAL INTEGRITY ASSESSMENTS Numerical creep-fatigue evaluation}

Since the principal goal of the research is the formulation of parametric relations able to describe long-term structural integrity of weldments, the creep-fatigue strength of the 5 configurations from Table 1 for type 1 and 2 weldments and 7 configurations with different $\rho$ values for type 3 weldments should be evaluated in a wide range of loading conditions. These conditions are presented by different combinations of $\Delta \varepsilon_{\text {tot }}$ in the parent plate outer fibre, as a characteristic of fatigue effects, and duration $\Delta t$ of dwell period, as a characteristic of creep effects. The set of 5 values for $\Delta \varepsilon_{\text {tot }}$ is the same as in the experimental studies [5,6]. The set of $\Delta t$ values used are the same as in the previous simulation study [3]: $0,0.5,1,2,5,10,100,1000$ and 10000 hours. Therefore, for each of configuration 45 creepfatigue evaluations must be performed with different values of $\Delta \varepsilon_{\text {tot }}$ and $\Delta t$. In order to estimate all values of number of cycles to failure $N^{\star}$, hundreds FE-simulations of the parametric models shown in Fig. 2 have been carried out, using the LMM method, material models and constants given in [3].
The concept of the proposed creep-fatigue evaluation procedure, considering time fraction rule for creep-damage assessment, is explained in detail in [3] and consists of 5 steps:

1. Estimation of saturated hysteresis loop using the LMM;

2. Estimation of fatigue damage using S-N diagrams;

3. Assessment of stress relaxation with elastic follow-up;

4. Estimation of creep damage using creep rupture curves;

5. Estimation of total damage using an interaction diagram.

Since the LMM requires lower computational effort compared to other methods, it appears to be an effective tool for express analysis of a large number of different loading cases using automation techniques. In order to perform hundreds FEsimulations in CAE-system ABAQUS and effectively retrieve corresponding values of $N^{\star}, 3$ analysis improvements using automation have been developed and in these parametric studies.

The first automation technique is the embedding of all 5 steps of the proposed creep-fatigue evaluation procedure in FORTRAN code of user material subroutine UMAT containing the implementation of the LMM and material models described in [3]. The most important parameters (derived in the 1st step of the procedure) for further creep-fatigue evaluation are the total strain range $\Delta \varepsilon_{\text {tot }}$, stress $\sigma_{1}$ at the beginning of dwell period and the elastic follow-up factor $Z$. These parameters from each integration point with material properties for elasticity, fatigue and creep, defined in the ABAQUS input file, are transferred into a new subroutine. This subroutine implements the next 4 steps of the procedure [3], which calculates and outputs the following parameters into ABAQUS result ODB-file: time to creep rupture $t^{*}$, creep damage accumulated per cycle $\omega_{1 \mathrm{c}}^{\mathrm{cr}}$, number of cycles to fatigue failure $N^{*}$, fatigue damage accumulated per 1 cycle $\omega_{1 \mathrm{c}}^{\mathrm{f}}$, and the most important - total number of cycles to creep-fatigue failure $N^{\star}$ based upon a damage interaction diagram.

The detailed description of this technique in application to FEA-results of type 1 and 2 weldments is reported in [11]. An example of the creep-fatigue evaluation procedure outputs for the configuration no. 2 (typically dressed) of type 2 weldment corre- 


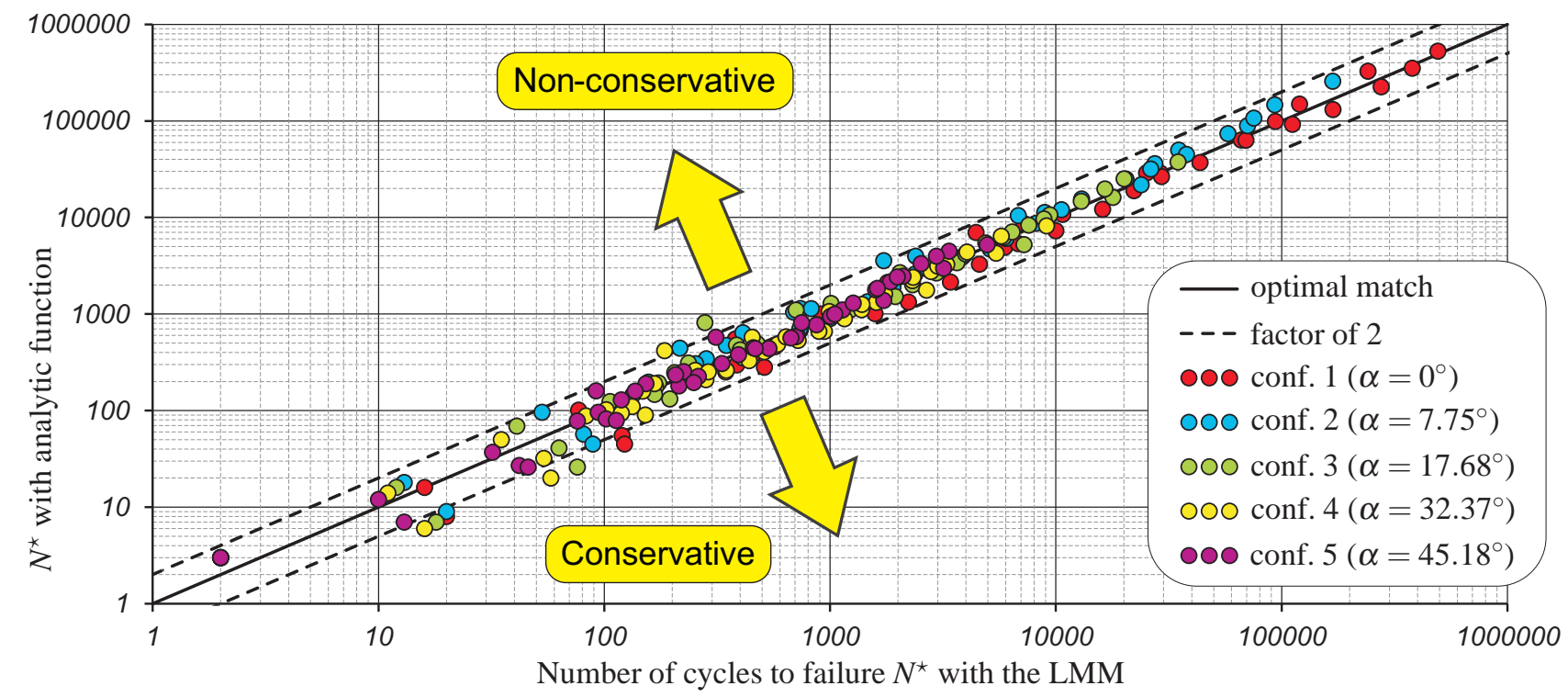

FIG. 3 COMPARISON OF $N^{\star}$ OBTAINED WITH THE LMM AND THE ANALYTIC FUNCTION 10) FOR TYPE 1 WELDMENT

sponding to the loading case of $\Delta \varepsilon_{\text {tot }}=1 \%$ and $\Delta t=5$ hours is presented in [11] and illustrated there in Fig. 5. Those results correspond to the FEA contour plots of the LMM outputs (obtained in Step 1) including $\Delta \varepsilon_{\mathrm{tot}}, \varepsilon^{\mathrm{cr}}, \varepsilon_{\mathrm{vM}}^{\mathrm{eq}}$ at the beginning of dwell and $\varepsilon_{\mathrm{vM}}^{\mathrm{eq}}$ at the end of dwell, explained in [3] and illustrated there in Fig. 9. The critical location with $N^{\star}=279$ cycles to failure for this case is the corner element in the weld toe adjacent to HAZ. Exactly the same approach is used to demonstrate an example of a type 1 weldment comprising geometry configuration no. 2 (typically dressed) and loading case of $\Delta \varepsilon_{\mathrm{tot}}=1 \%$ and $\Delta t=5$ hours. Figure 6 in [11] shows the outputs of FEA with the LMM, while Fig. 7 in [11] shows the outputs of the creep-fatigue evaluation procedure. The critical location with $N^{\star}=206$ cycles to failure for type 1 is the same as for the type 2 weldment - the corner element in the weld toe adjacent to HAZ. The examples of FEA results for type 3 weldment under the same loading conditions and their analysis are to be presented at the conference.

The second automation technique is the development of a stand-alone application using Embarcadero Delphi integrated development environment using Delphi programming language. This simple application automatically carries out the sequence of all FE-simulations with different $M$ (corresponding to $5 \Delta \varepsilon_{\text {tot }}$ values) and $\Delta t$ values for each of the configurations of type 1 , 2 and 3 weldments. This is implemented by automated modification of the UMAT subroutine including changing of loading values ( $M$ and $\Delta t$ ) and output file names, therefore producing individual ABAQUS result ODB-file for each loading case.

The third automation technique is the development of a script using ABAQUS Python Development Environment (Abaqus PDE) using Python programming language [12]. This simple script, when started in ABAQUS/CAE environment, appends the list of available ABAQUS result ODB-files corresponding to one configuration. For each of ODB-files, it reads the values of $N^{\star}$ in each integration point, selects the integration point with minimum value of $N^{\star}$ over the FE-model, and writes the element number, integration point number and material name to an output text file. Therefore, the critical locations and corresponding values of $N^{\star}$ are extracted automatically for all configurations and loading cases. Obtained results can be used for the formulation of an analytic assessment model suitable for the fast estimation of $N^{\star}$ for a variety of loading conditions $(\tilde{M}$ and $\Delta t)$ and geometrical weld profile parameters $(\alpha, \beta$ and $\rho)$.

\section{Analytic assessment model}

For each of the configurations for type 1, 2 and 3 weldments, the array of assessment results consisting of $N^{\star}$ values corresponding to particular values of $\tilde{M}$ and $\Delta t$ is fitted using the least squares method by the following function proposed in the form of power-law in [3]:

$$
\log \left(N^{\star}\right)=\tilde{M}^{-b(\Delta t)} / a(\Delta t),
$$

where the fitting parameters dependent on dwell period $\Delta t$ are

$$
\begin{aligned}
a(\Delta t)= & a_{3} \log (\Delta t+1)^{3}+a_{2} \log (\Delta t+1)^{2} \\
& +a_{1} \log (\Delta t+1)+a_{0} \quad \text { and } \\
b(\Delta t)= & b_{3} \log (\Delta t+1)^{3}+b_{2} \log (\Delta t+1)^{2} \\
& +b_{1} \log (\Delta t+1)+b_{0},
\end{aligned}
$$


and the independent fitting parameters $\left(a_{0}-a_{3}\right.$ and $\left.b_{0}-b_{3}\right)$ have particular values individual for each type of weldment (1,2 and 3 ) and each available configuration.

In order to capture all configurations with an unified set of fitting parameters, parameters $a_{0}, a_{1}, a_{2}, a_{3}, b_{0}, b_{1}, b_{2}, b_{3}$ should be defined as dependent on geometric parameters $\alpha, \beta$ and $\rho$ using the least squares method. For the type 1 weldments these parameters are dependent on angle $\alpha$ only:

$$
\begin{aligned}
& a_{0}^{\mathrm{T} 1}(\alpha)=-4.175 \cdot 10^{-5} \alpha^{2}+2.72 \cdot 10^{-3} \alpha+0.227 \\
& a_{1}^{\mathrm{T} 1}(\alpha)=-2.169 \cdot 10^{-3} \alpha+1.21 \cdot 10^{-1} \\
& a_{2}^{\mathrm{T} 1}(\alpha)=1.907 \cdot 10^{-3} \alpha-7.093 \cdot 10^{-2} \\
& a_{3}^{\mathrm{T} 1}(\alpha)=-5.352 \cdot 10^{-4} \alpha+1.968 \cdot 10^{-2} \\
& b_{0}^{\mathrm{T} 1}(\alpha)=-4.76324 \cdot 10^{-3} \alpha+0.793 \\
& b_{1}^{\mathrm{T} 1}(\alpha)=1.42 \cdot 10^{-4} \alpha^{2}-8.547 \cdot 10^{-3} \alpha+0.4028 \\
& b_{2}^{\mathrm{T} 1}(\alpha)=1.531 \cdot 10^{-3} \alpha-0.3015 \\
& b_{3}^{\mathrm{T} 1}(\alpha)=-3.08 \cdot 10^{-4} \alpha+8.364 \cdot 10^{-2}
\end{aligned}
$$

For the type 2 weldments these parameters include the dependence on angle $\alpha$ from Eqs (12) and an additional effect of angle $\beta$ as in the following form:

$$
\begin{aligned}
a_{0}^{\mathrm{T} 2}(\alpha, \beta)= & a_{0}^{\mathrm{T} 1}(\alpha)+3.179 \cdot 10^{-4} \beta+2.355 \cdot 10^{-3}, \\
a_{1}^{\mathrm{T} 2}(\alpha, \beta)= & a_{1}^{\mathrm{T} 1}(\alpha)-1.636 \cdot 10^{-3} \beta+3.043 \cdot 10^{-2}, \\
a_{2}^{\mathrm{T} 2}(\alpha, \beta)= & a_{2}^{\mathrm{T} 1}(\alpha)+1.636 \cdot 10^{-3} \beta-3.043 \cdot 10^{-2}, \\
a_{3}^{\mathrm{T} 2}(\alpha, \beta)= & a_{3}^{\mathrm{T} 1}(\alpha)-4.136 \cdot 10^{-4} \beta+7.33 \cdot 10^{-3}, \\
b_{0}^{\mathrm{T} 2}(\alpha, \beta)= & b_{0}^{\mathrm{T} 1}(\alpha)+0.0291 \\
& -1.684 \cdot 10^{-4} \exp (0.1622 \beta), \\
b_{1}^{\mathrm{T} 2}(\alpha, \beta)= & b_{1}^{\mathrm{T} 1}(\alpha)-0.1789 \\
b_{2}^{\mathrm{T} 2}(\alpha, \beta)= & b_{2}^{\mathrm{T} 1}(\alpha)+0.1558 \\
b_{3}^{\mathrm{T} 2}(\alpha, \beta)= & b_{3}^{\mathrm{T} 1}(\alpha)-4.546 \cdot 10^{-2} .
\end{aligned}
$$

For the type 3 weldments these parameters are dependent on ratio $\rho$ only in the following form:

$$
\begin{aligned}
& a_{0}^{\mathrm{T} 3}(\rho)=-4.506 \cdot 10^{-2} \ln (\rho+1)+0.285, \\
& a_{1}^{\mathrm{T} 3}(\rho)=4.1 \cdot 10^{-2} \ln (\rho+1)+4.701 \cdot 10^{-2}, \\
& a_{2}^{\mathrm{T} 3}(\rho)=-3.202 \cdot 10^{-2} \ln (\rho+1)-7.575 \cdot 10^{-3}, \\
& a_{3}^{\mathrm{T} 3}(\rho)=8.74 \cdot 10^{-3} \ln (\rho+1)+2.10773 \cdot 10^{-3} \\
& b_{0}^{\mathrm{T} 3}(\rho)=0.118 \ln (\rho+1)+0.57 \\
& b_{1}^{\mathrm{T} 3}(\rho)=8.742 \cdot 10^{-2} \ln (\rho+1)+0.195, \\
& b_{2}^{\mathrm{T} 3}(\rho)=-7.197 \cdot 10^{-2} \ln (\rho+1)-0.152, \\
& b_{3}^{\mathrm{T} 3}(\rho)=1.397 \cdot 10^{-2} \ln (\rho+1)+4.034 \cdot 10^{-2} .
\end{aligned}
$$

The verification of the fit quality using the the geometrical parameters $(\alpha, \beta$ and $\rho)$ for the proposed relations (12) - (14) is implemented by applying Eqs (10) and (11) to estimate $N^{\star}$. Number of cycles to failure $N^{\star}$ is estimated for all configurations using the corresponding values of angles ( $\alpha$ and $\beta$ ) from Table 1 and ratio $\rho(2.0,1.5,1.0,0.5,0.2,0.1$ and $\rightarrow 0)$. The comparison is done for the same load combinations as were used for the LMM analyses. The results of the verification are illustrated on diagrams in Fig. 3 for type 1, Fig. 4 for type 2 and Fig. 5 for type 3 weldments in the form of $N^{\star}$ obtained with the analytic function (10) vs. $N^{\star}$ obtained with the LMM. Comparison of the analytic and numeric $N^{\star}$ for all types of weldments shows that the quality of analytic predictions is quite close to the line of optimal match and provides a uniform scatter of results through all variants of loading conditions and configurations. The discrepancy between analytic predictions and numerical LMM outputs is generally found to be within the boundaries of an inaccuracy factor equal to 2, which is allowable for engineering analysis, producing both conservative and non-conservative results.

Having defined the number of cycles to failure $N^{\star}$ by Eq. (10), the residual service life in years is therefore dependent on the duration of 1 cycle, which consists of dwell period $\Delta t$ and relatively short time of deformation as follows:

$$
L^{\star}=N^{\star}\left[\frac{\Delta t}{365 \cdot 24}+\frac{2 \Delta \varepsilon_{\mathrm{tot}}(\tilde{M})}{\dot{\varepsilon}(365 \cdot 24 \cdot 60 \cdot 60)}\right]
$$

where $\dot{\varepsilon}=0.03 \% / \mathrm{s}$ is a strain rate according to experimental conditions [5, 6], and the parametric analytical relations for $\Delta \varepsilon_{\mathrm{tot}}(\tilde{M})$ are derived in Sect. 3 of [11]. These relations for $\Delta \varepsilon_{\text {tot }}(\tilde{M})$ include the geometrical parameters of parent plate cross-section (thk and $w)$ and weld profile $(\alpha, \beta$ and $\rho)$, and parent plate material parameters $\left(E, v, B, \beta, \sigma_{\mathrm{y}}\right)$.

\section{PARAMETRIC FORMULATION OF FSRF}

Since the function $N^{\star}(\tilde{M}, \Delta t)$ proved its validity in the previous subsection, it can be applied for the fast creep-fatigue assessments of new welded structures during the design stage. However, it is generally hard to generate conclusions about the service conditions $(\tilde{M}, \Delta t)$ required to estimate particular value of $N^{\star}$. Loading conditions comprise a wide range of mechanical loading described by $\tilde{M}$ or corresponding range of $\Delta \varepsilon_{\text {tot }}$ in parent material adjacent to welded joints. Thus, introduction of a Fatigue Strength Reduction Factor (FSRF) allows a wide range of mechanical loading relevant to application area of a designed welded structure to be captured. The FSRF takes into account the difference in behaviour of the weldment compared to the parent material, considering weldments to be composed of parent material. The FSRF is determined experimentally by comparing the fatigue failure data of the welded specimen with the fatigue curve derived from tests on the parent plate material.

The current approach in R5 Volume 2/3 Procedure [7] operates with the fixed values of FSRF for 3 different types of 


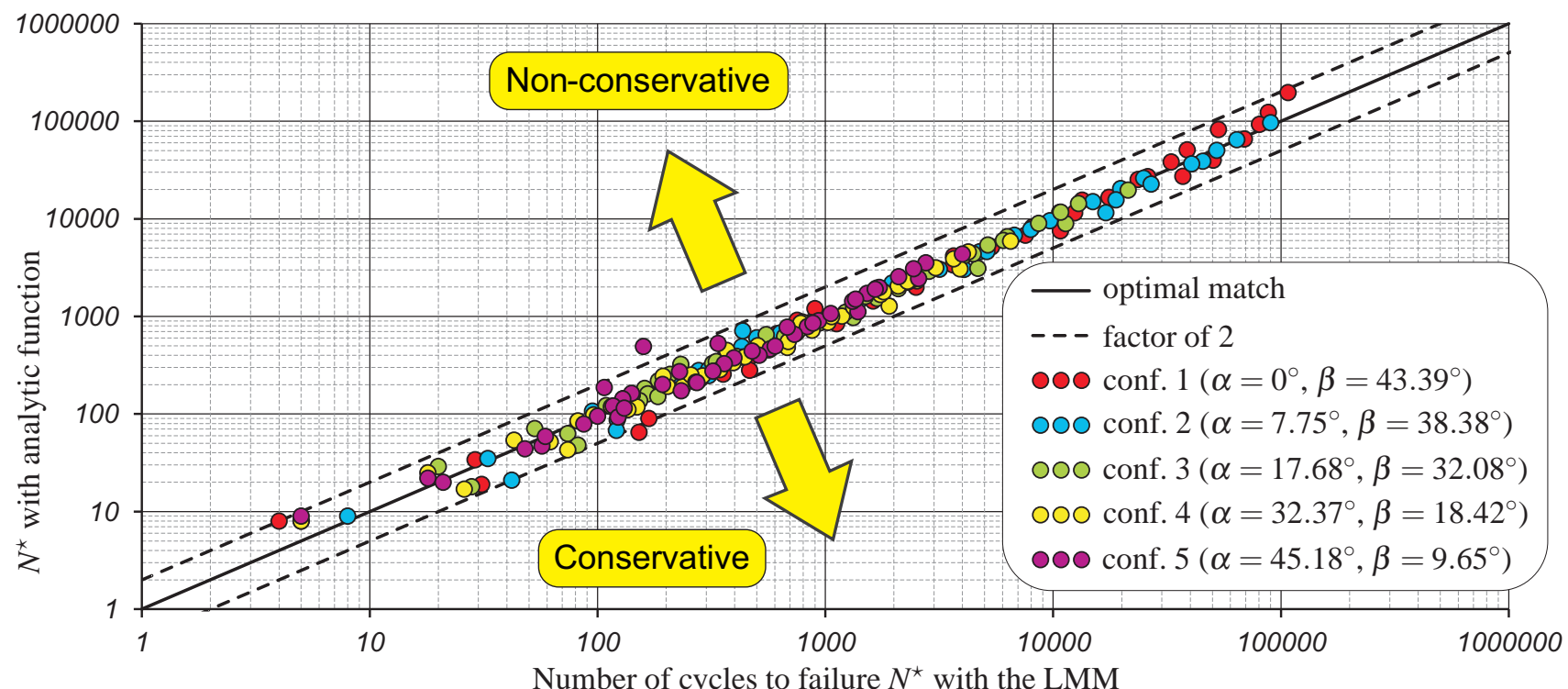

FIG. 4 COMPARISON OF $N^{\star}$ OBTAINED WITH THE LMM AND THE ANALYTIC FUNCTION (10) FOR TYPE 2 WELDMENT

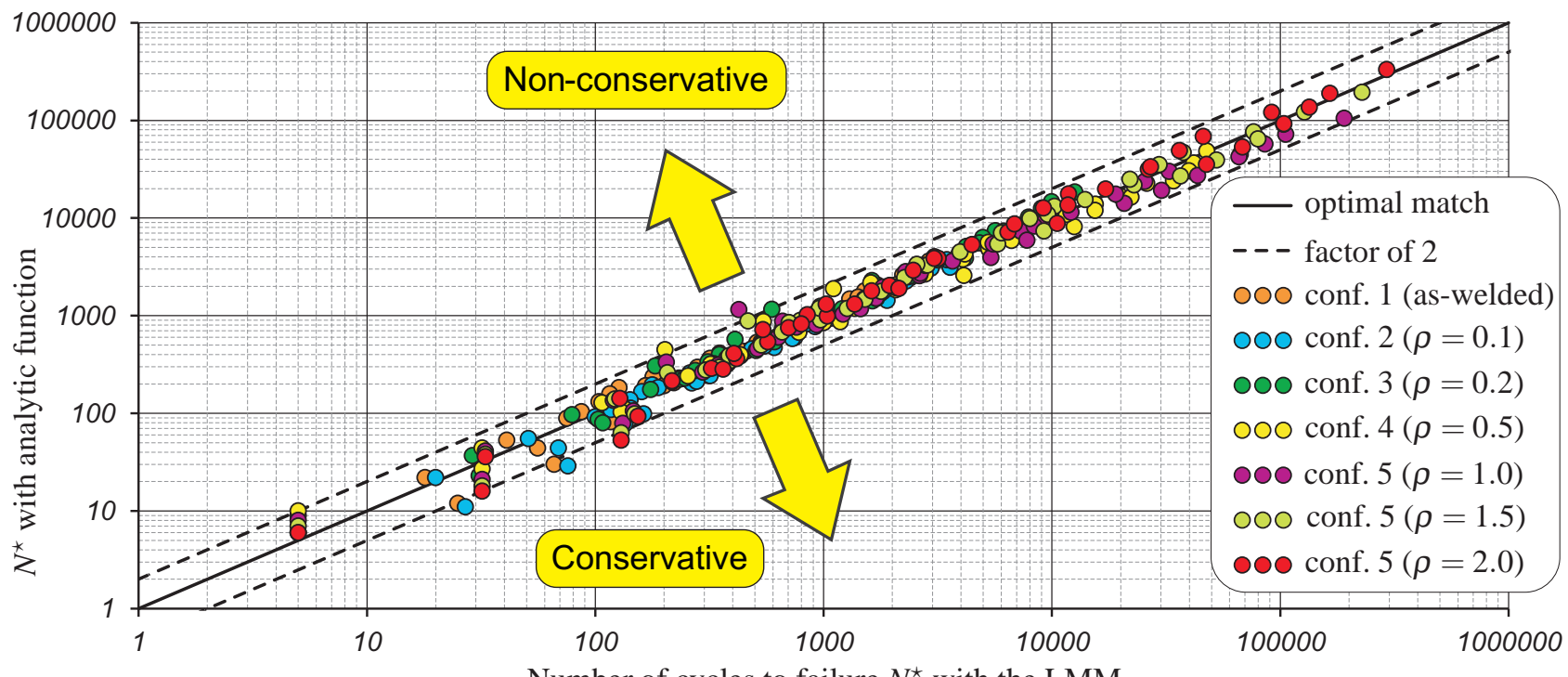

Number of cycles to failure $N^{\star}$ with the LMM

FIG. 5 COMPARISON OF $N^{\star}$ OBTAINED WITH THE LMM AND THE ANALYTIC FUNCTION (10) FOR TYPE 3 WELDMENT

weldments taking into account dressed and as-welded variants, which consider only the reduction of fatigue strength of weldments compared to the parent material. For austenitic steel weldments [13], FSRF $=1.5$ is prescribed for both variants of type $1, \mathrm{FSRF}=1.5$ for type 2 dressed and FSRF $=2.5$ for as-welded variant, and FSRF $=3.2$ is prescribed for both variants of type 3 . All this variety of the FSRFs is representative of the reduction in fatigue endurance caused by the local strain range $\varepsilon_{\text {tot }}$ enhancement in the weldment region due to the material discontinuity and geometric strain concentration effects. The introduction of
FSRF as dependent on $\Delta t$ in [3] using function $N^{\star}(\tilde{M}, \Delta t)$ for the case of type 2 dressed weldment allowed the influence of creep to be taken into account, and to provide the adjusted values of FSRF for creep-fatigue operation conditions. Therefore, the same approach [3] is applied to obtain $\Delta t$-dependent FSRFs for a variety of geometrical configurations considering additional dependence on parameters of weld profile $(\alpha, \beta$ and $\rho)$.

For this purpose Eq. 10 is converted analytically to the relation $\tilde{M}\left(N^{\star}, \Delta t\right)$ and inserted into the group of relations $\Delta \varepsilon_{\text {tot }}(\tilde{M})$ as explained in [11], resulting in the relation 


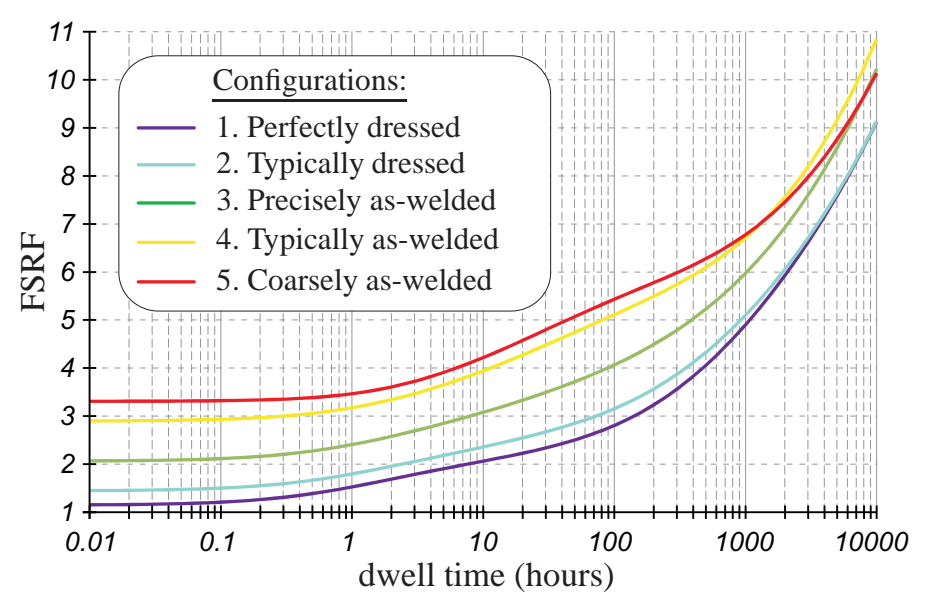

FIG. 6 FSRFS DEPENDENT ON DWELL PERIOD $\Delta t$ FOR DIFFERENT CONFIGURATIONS OF TYPE 1 WELDMENTS

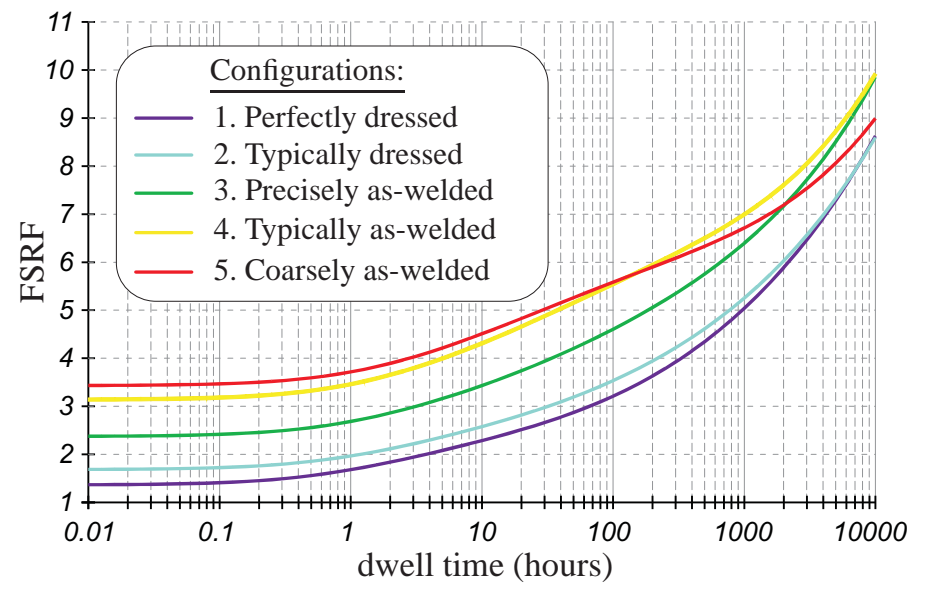

FIG. 7 FSRFS DEPENDENT ON DWELL PERIOD $\Delta t$ FOR DIFFERENT CONFIGURATIONS OF TYPE 2 WELDMENTS

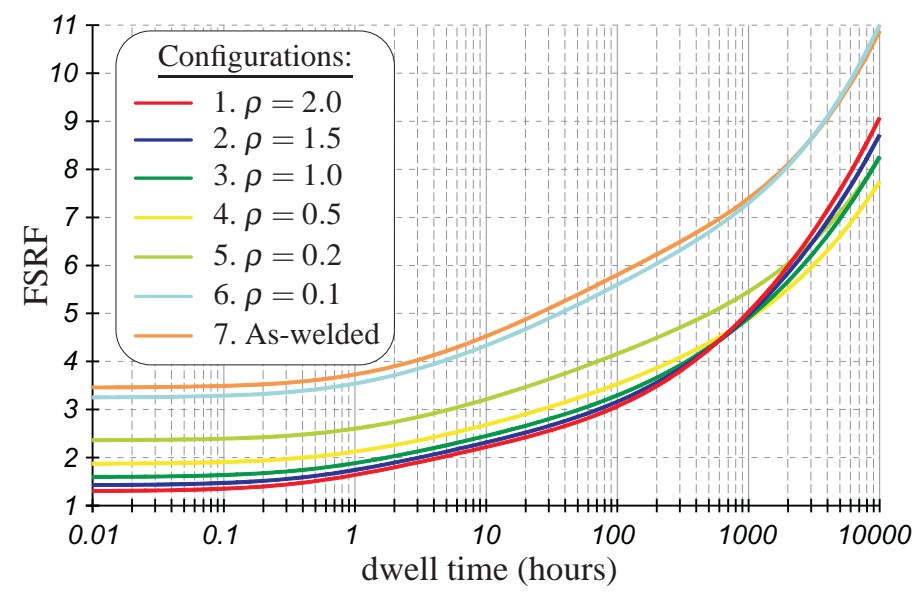

FIG. 8 FSRFS DEPENDENT ON DWELL PERIOD $\Delta t$ FOR DIFFERENT CONFIGURATIONS OF TYPE 3 WELDMENTS
TABLE 2 THE VALUES OF FSRFS FOR PURE FATIGUE FOR TYPES 1, 2 AND 3 WELDMENTS FROM FIGS 68

\begin{tabular}{c|ccccccc}
\hline \hline Conf. & 1 & 2 & 3 & 4 & 5 & 6 & 7 \\
\hline Type 1 & 1.146 & 1.444 & 2.062 & 2.896 & 3.308 & - & - \\
Type 2 & 1.362 & 1.682 & 2.372 & 3.137 & 3.430 & - & - \\
Type 3 & 1.302 & 1.425 & 1.595 & 1.872 & 2.362 & 3.252 & 3.459 \\
\hline \hline
\end{tabular}

$\Delta \varepsilon_{\text {tot }}\left(N^{\star}, \Delta t,[\alpha, \beta\right.$ or $\left.\rho]\right)$. This relation describes the $\Delta \varepsilon_{\text {tot }}$ in the parent material remote from weldment corresponding to particular values of $N^{\star}$ and $\Delta t$ for a particular geometrical configuration of weldment defined by $\alpha, \beta$ or $\rho$. Thus, the FSRFs, appropriate to varying values of $\Delta t$ and equal values of $N^{\star}$, are defined by the relation between the $\mathrm{S}-\mathrm{N}$ diagram corresponding to fatigue failures of parent material plate and S-N diagrams for a weldment:

$$
\mathrm{FSRF}=\Delta \varepsilon_{\mathrm{tot}}^{\mathrm{par}}\left(N^{\star}\right) / \Delta \varepsilon_{\mathrm{tot}}\left(N^{\star}, \Delta t,[\alpha, \beta \text { or } \rho]\right),
$$

where the $\mathrm{S}-\mathrm{N}$ diagram for parent material plate is defined as

$$
\log \left(\Delta \varepsilon_{\mathrm{tot}}^{\mathrm{par}}\right)=p_{0}+p_{1} \log \left(N^{*}\right)+p_{2} \log \left(N^{*}\right)^{2},
$$

with the following polynomial coefficients referring to [13]: $p_{0}=2.2274, p_{1}=-0.94691$ and $p_{2}=0.085943$.

The FSRFs estimated by Eq. (16) corresponding to the range of $\Delta t \in\left[0 \ldots 10^{5}\right]$ hours are defined in some particular range of $N^{\star}$. This range is different for each value of $\Delta t$ characterised by reducing value of the average $N^{\star}$ with the growth of $\Delta t$. The upper bound of the $N^{\star}$ range is governed by the mathematical upper limit of the S-N diagram $\Delta \varepsilon_{\text {tot }}^{\mathrm{par}}\left(N^{\star}\right)$ for parent material plate, which is defined in [3] as $\log \left(N_{\max }^{\star}\right)=p_{1} /\left(2 p_{2}\right)=5.51$ or $\Delta \varepsilon_{\mathrm{tot}}^{\mathrm{par}}\left(10^{5.51}\right)=0.416 \%$. The lower bound of the $N^{\star}$ range is flexible and governed by $\Delta t$ using the following function:

$$
\log \left(N_{\min }^{\star}\right)=3-0.5 \log (\Delta t+1) .
$$

Finally, for all proposed configurations of all weld types the FSRF is defined as a continuous function of $\Delta t$ using Eq. (16) using simple averaging procedure over a dynamic range of $N^{\star}$ from $\log \left(N_{\min }^{\star}\right)$ to $\log \left(N_{\max }^{\star}\right)$ with step 0.01 . The resultant dependencies of FSRFs on $\Delta t$ are illustrated in Fig. 6 for type 1, Fig. 7 for type 2, and Fig. 8 for type 3 weldments with designation of different configurations. First of all, these figures show significant enhancement of FSRF for dwells $\Delta t>0.1$ hour caused by creep, which is important for design applications. The initial values of FSRFs corresponding to pure fatigue conditions $(\Delta t=0)$ are listed in Table 2 and could be compared with the values recommended in R5 Volume 2/3 Procedure [7]. 
The FSRF for type 1 dressed weldments is within the range 1.146-1.444 depending on the quality of grinding, while R5 gives the value 1.5 (refer to [13]), which is more conservative. The FSRF for type 1 precisely welded joints without grinding is within the range 1.444-2.062 depending on the quality of welding, while R5 gives the same value 1.5, which is nonconservative. The FSRF for type 1 coarsely welded joints without any additional treatment may reach up to 3.308 , while R5 doesn't give any value for this case.

The FSRF for type 2 dressed weldments is within the range 1.362-1.682 depending on the quality of grinding, while R5 gives the value 1.5 , which approximately corresponds to average value for the obtained range. The FSRF for type 2 precisely welded joints without grinding is within the range 1.682-2.372 depending on the quality of welding, while R5 gives the value 2.5 , which is more conservative. The FSRF for type 2 coarsely welded joints without any additional treatment may reach up to 3.43, while R5 doesn't give any value for this case.

The FSRF for type 3 dressed weldments is within the range $1.302-1.425$, for type 3 welded joints with moderate TIG dressing it is within the range 1.425-2.362 depending on the amount of TIG dressing, while R5 also doesn't give any value for these cases. The FSRF for type 3 as-welded joints without any additional treatment may reach up to 3.252-3.459, while R5 gives the value 3.2 , which approximately corresponds to lower bound for the obtained range. It should be noted that the value of FSRF for type 3 recommended by R5 procedure may be significantly conservative, if some kind of TIG dressing is applied.

\section{CONCLUSIONS}

The parametric study on creep-fatigue strength of the steel AISI type $316 \mathrm{~N}(\mathrm{~L})$ weldments of types 1,2 and 3 according to classification of R5 Vol. 2/3 procedure [7] at $550^{\circ} \mathrm{C}$ has been implemented using the LMM. The study is based upon the latest developed creep-fatigue evaluation procedure [3] considering time fraction rule for creep-damage assessment. Proposed approach improves upon existing design techniques, e.g. in R5 procedure [7], by considering the significant influence of creep. Moreover, the obtained FSRFs for pure fatigue revises the values recommended in R5 Procedure [7] removing the redundant conservatism for type 1 and 3 dressed weldments and type 2 undressed weldments.

\section{ACKNOWLEDGMENT}

The authors deeply appreciate the Engineering and Physical Sciences Research Council (EPSRC) of the UK for the financial support in the frames of research grant no. EP/G038880/1, the University of Strathclyde for hosting during the course of this work, and EDF Energy for the experimental data.

\section{REFERENCES}

[1] Lee, Y.-L., Barkey, M. E., and Kang, H.-T., 2012. Metal Fatigue Analysis Handbook: Practical Problem-Solving Techniques for Computer-Aided Engineering. ButterworthHeinemann, Oxford.

[2] Radaj, D., Sonsino, C. M., and Fricke, W., 2006. Fatigue Assessment of Welded Joints by Local Approaches, 2nd ed. Woodhead Publishing Limited, Cambridge.

[3] Gorash, Y., and Chen, H., 2013. "Creep-fatigue life assessment of cruciform weldments using the linear matching method". Int. J. of Pressure Vessels \& Piping. in press, DOI: 10.1016/j.ijpvp.2012.12.003.

[4] Chen, H. F., Chen, W., and Ure, J., 2012. "Linear matching method on the evaluation of cyclic behaviour with creep effect". In Proc. ASME Pressure Vessels \& Piping Conf. (PVP2012), ASME. July 15-19.

[5] Bretherton, I., and Budden, P. J., 1999. "Assessment of creep-fatigue endurance of large cruciform weldments". In Trans. 15th Int. Conf. on Structural Mechanics in Reactor Technology, no. SMiRT15 - F05/2. IASMiRT, Seoul, Korea, pp. 185-192.

[6] Bretherton, I., Knowles, G., Hayes, J.-P., Bate, S. K., and Austin, C. J., 2004. PC/AGR/5087: Final report on the fatigue and creep-fatigue behaviour of welded cruciform joints. Report for British Energy Generation Ltd no. RJCB/RD01186/R01, Serco Assurance, Warrington, UK.

[7] Ainsworth, R. A., ed., 2003. R5: An Assessment Procedure for the High Temperature Response of Structures. Procedure R5: Issue 3. British Energy Generation Ltd, Gloucester, UK.

[8] British Standard, 2010. Welding - Basic welded joint details in steel - Part 1: Pressurized components. No. EN 17081:2010. London, UK.

[9] British Standard, 2007. Welding - Fusion-welded joints in steel, nickel, titanium and their alloys - Quality levels for imperfections. No. EN ISO 5817:2007. London, UK.

[10] Pedersen, M. M., Mouritsen, O. O., Hansen, M. R., Andersen, J. G., and Wenderby, J., 2010. "Comparison of post weld treatment of high strength steel welded joints in medium cycle fatigue". Welding in the World, 54(7-8), pp. R208-R217.

[11] Gorash, Y., and Chen, H., 2013. "A parametric study on creep-fatigue strength of welded joints using the linear matching method". Int. J. of Fatigue. submitted, no. IJFATIGUE-D-13-00028, StrathPrints URI

[12] Dassault Systèmes Simulia CoRP., 2010. ABAQUS Scripting User's Manual, Version $6.10 \mathrm{ed}$.

[13] Bate, S. K., Hayes, J.-P., Hooton, D. G., and Smith, N. G., 2005 . Further analyses to validate the R5 volume $2 / 3$ procedure for the assessment of austenitic weldments. Report for British Energy Generation Ltd no. SA/EIG/11890/R002, Serco Assurance, Warrington, UK. 